Schwerpunkt

Gastroenterologe 2020 15:487-493 https://doi.org/10.1007/s11377-020-00481-4 Online publiziert: 28 . Oktober 2020 (c) Springer Medizin Verlag $\mathrm{GmbH}$, ein Teil von Springer Nature 2020

\section{Redaktion}

S. Ciesek, Frankfurt am Main

J. F. Riemann, Ludwigshafen

\author{
M. Banysch ${ }^{1}$ T. Heuer ${ }^{2}$ G. Frings ${ }^{3} \cdot$ H. Kühl ${ }^{4} \cdot$ S. Bochlogyros ${ }^{1,5} \cdot$ G. M. Kaiser ${ }^{1}$ \\ 'Klinik für Allgemein- und Viszeralchirurgie, St. Bernhard-Hospital Kamp-Lintfort, Kamp-Lintfort, \\ Deutschland \\ ${ }^{2}$ Medizinische Klinik I, St. Bernhard-Hospital, Kamp-Lintfort, Deutschland \\ ${ }^{3}$ Klinik für Anästhesie und Intensivmedizin, St. Bernhard-Hospital Kamp-Lintfort, Kamp-Lintfort, \\ Deutschland \\ ${ }^{4}$ Klinik für Radiologie, St. Bernhard-Hospital Kamp-Lintfort, Kamp-Lintfort, Deutschland \\ ${ }^{5}$ Corona-Screening-Center, St. Bernhard-Hospital Kamp-Lintfort, Kamp-Lintfort, Deutschland
}

\title{
Herausforderungen der Viszeralmedizin bei COVID-19
}

\section{Erfahrungen aus einem Krankenhaus der Grund- und Regelversorgung}

die Absage elektiver Eingriffe v.a. von März bis Mai 2020, deutlich erweitert. Auch für den Bereich der Viszeralmedizin sind spezifische Maßnahmen ergriffen worden, um die Verbreitung des Virus zu verhindern oder zumindest zu verlangsamen.

) Die Mortalität von COVID19 wird je nach Ursprungsland und Aufarbeitungsmethode sehr variabel angegeben

Die Mortalität von COVID-19 wird in der Literatur je nach Ursprungsland und Aufarbeitungsmethode sehr variabel angegeben und beträgt nach Angaben der WHO im Mittel 4,5 \% [20]. Zu den Hochrisikopatienten zählen ältere Patienten, onkologisch Kranke und andere Patientengruppen, die eine immunsuppressive Therapie erhalten (etwa Transplantierte/Patienten mit chronisch-entzündlichen Darmerkrankungen). Risikogruppen bedürfen einer besonders intensiven Prävention, Kontrolle und Behandlung, wie dies auch schon bei anderen Viruserkrankungen wie der „Schweinegrippe“ beschrieben wurde $[4,18]$.

Trotzaller gebotenen Umsicht gilt weiterhin, dass für Patienten mit nachgewiesener COVID-19-Infektion alle notwendigen diagnostischen und therapeu- tischen Verfahren zur Verfügung stehen müssen, auch wenn dies für alle Beteiligten einen großen logistischen Aufwand bedeutet [11].

Wegen der besonderen Situation wurde im St. Bernhard-Hospital Kamp-Lintfort ein Krisenstab gegründet, der anfangs täglich 2 Sitzungen abhielt. Die enge Kommunikation erfolgte mit dem Direktorium zusätzlich ab Mitte März über 3 Klinikkonferenzen pro Woche in 2 Gruppen für die leitenden Mitarbeiter des Krankenhauses, ab Juni finden diese wöchentlich statt. Die dargestellte Übersicht zeigt die für das Krankenhaus erfolgten Maßnahmen, die im Rahmen der COVID-19-Pandemie umgesetzt wurden (•Tab. 1).

\section{COVID-Screening-Center (CSC)}

Alle Patienten und Besucher können das Krankenhaus seit dem17.03.2020 nur mit Genehmigung und nach Durchlaufen des CSC betreten (• Abb. 1). Bei Stufe I erfolgen zunächst die Evaluation der Anamnese und eine Temperaturmessung (- Abb. 2). Auffällige Befunde werden in Stufe II immer im Behandlungszelt des CSC unter Berücksichtigung der Hygieneanforderungen weitergehend untersucht. Die hierzu korrespondierende Anzahl von Vorstellungen im CSC ist in - Abb. 3 dargestellt. Bei ambulanter Behandlungsmöglichkeit werden im teten Infektionen wurden die intensivmedizinischen Kapazitäten, auch durch 


\section{Schwerpunkt}

Tab. 1 Maßnahmen nach Bekanntwerden der COVID-19( „, coronavirus disease 2019")-Pandemie

\section{Strukturelle}

Änderungen

Patientenorientierte Anforderungen

Mitarbeiterorientierte Anforderungen

\section{Gründung COVID-Screening-Center mit Container und Zelt} te Fälle), Belegung 20 Patienten (weitere 20 bei Bedarf)

Aufrüstung der Intensivstation von 10 auf 20 Betten mit Beatmungsmöglichkeit

Getrennte Eingänge für Patienten, Besucher und Mitarbeiter

Cafeteria nur noch für Mitarbeiter zugänglich

Öffentliche Desinfektionsmittelspender im Eingangsbereich, in den

Fluren, vor jedem Zimmer

Hinweisschilder zu Verhaltensregeln in der Pandemie

Unterstützung durch externen Sicherheitsdienst

Treffen des Krisenstabs täglich bis Juni, danach bedarfsorientiert 1 bis 2 Termine/Woche

Besuchersperre und Beschränkungen (bei Bedarf und nach Genehmigung $1 \mathrm{~h} / 1$ Besucher pro Tag/Patient, Ausnahme: Sonderregelungen für palliative und sterbende Patienten)

Screening für alle Patienten und Besucher vor Betreten des Krankenhauses

Abstriche bei elektiven Aufnahmen von Patienten positiven Befunden von COVID-19 und Verdachtsfällen

Persönliche Schutzausrüstung nach Risiko
Etablierung einer Isolationsstation (Trennung Verdachts- und bestätig-

Isolierung auf der Infektionsstation und in den Funktionsbereichen bei

Maskenpflicht (Stoffmasken und deren Reinigung organisiert durch das Krankenhaus)

Tagebuch bei Kontaktpersonen mit Symptom- und Temperaturkontrolle

Kontrolle bei Reiserückkehrern aus Risikogebieten

Schulung im Umgang mit COVID-19

Fortbildungen an den Beatmungsgeräten

Information über Klinikkonferenzen an alle Mitarbeiter anfangs täglich

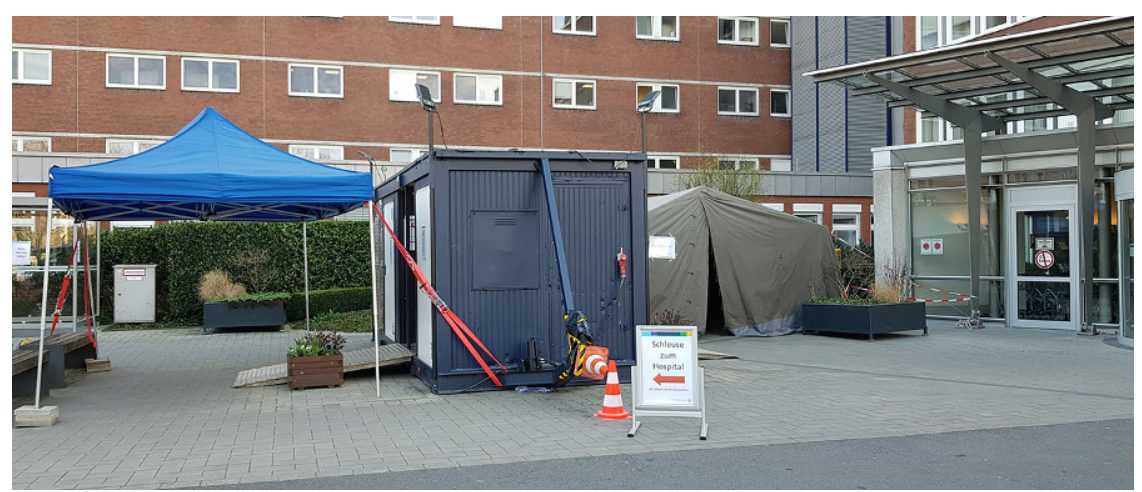

Abb. $1 \Delta$ Darstellung des Corona-Screening-Centers für das Personenscreening von Patienten und Besuchern vor dem Haupteingang des Krankenhauses, das als Provisorium im März 2020 eingerichtet wurde

Screening auffällige Patienten an den Hausarzt und das Gesundheitsamt verwiesen. Die Anzahl der nach Screening zugelassenen Besucher ist in - Abb. 4 gezeigt.

Patienten, die sich einer stationären Behandlung unterziehen müssen, wer- den seit Mai 2020 unabhängig von ihrer Symptomatik vor stationärer Aufnahme über einen Nasen- und Rachenabstich mit PCR („polymerase chain reaction“) auf das Virus getestet. Die elektiven Patienten werden angewiesen, ab dem Tag des Screenings in häuslicher Quarantäne zu bleiben, um eine zwischenzeitliche Infektion bis zur stationären Aufnahme zu vermeiden. Nach RKI-Empfehlung vom 11.08.2020 ist jedoch eine spezifische Testung für Patienten nach negativem Screening und Anamnese nicht zwingend erforderlich und das Vorgehen der Kliniken für ambulante und stationäre Patienten somit sehr unterschiedlich [14].

\section{Verhalten im Team der Viszeralmedizin}

Neben den persönlichen Schutzmaßnahmen sind im Krankenhaus mehrere Maßnahmen ergriffen worden, um die Exposition so gering wie möglich zu halten:

- Reduktion von ambulanten Sprechstunden;

- besondere Verhaltensregeln bei Visiten;

- Behandlungsteam so klein wie möglich;

- kurze Expositionszeit bei Patienten über eingespielte Teams;

- als Kontaktperson für Mitarbeiter gezielte Testung auf COVID-19 und Führen von Tagebuch zu allgemeiner Gesundheit und Fieber;

- Testung aller Ärzte des Krankenhauses auf COVID-19;

- negativer Befund gefordert nach Rückreise aus Risikogebieten vor Arbeitsbeginn;

- Ausbildungseingriffe müssen in der Pandemie tendenziell unterbleiben;

- Im Gipfel der Krise Vorhalten eines Ärzteteams außerhalb des Krankenhauses, um ein zweites Team stellen zu können bei Infektion des „VorOrt-Teams“.

Bei der interventionellen Endoskopie sowie bei der laparoskopischen- und offenen Chirurgie kann die Anwendung von Ultraschalldissektoren oder von monopolarem Strom gefährlichen Rauch entwickeln $[1,10]$. Vor allem die Aerosole des Ultraschalldissektors mit niedrigen Temperaturen können die infektiösen Komponenten des Virus nicht deaktivieren, sondern zu einer zusätzlichen Exposition mit dem Virus führen. In verschiedenen Studien sind multiple Viren wie Hepatitis-B- und Papillomaviren bereits im Rauch nachgewiesen worden $[7,18]$. 
Gastroenterologe 2020 15:487-493 https://doi.org/10.1007/s11377-020-00481-4

(c) Springer Medizin Verlag GmbH, ein Teil von Springer Nature 2020

\section{Banysch · T. Heuer · G. Frings $\cdot$ H. Kühl $\cdot$ S. Bochlogyros · G. M. Kaiser}

\section{Herausforderungen der Viszeralmedizin bei COVID-19. Erfahrungen aus einem Krankenhaus der Grund- und Regelversorgung}

\section{Zusammenfassung}

Hintergrund. Die sich weltweit ausbreitende COVID-19("coronavirus disease 2019")Pandemie hat sich innerhalb weniger Wochen auch in Deutschland zu einem zunehmenden Problem im Gesundheitssystem und in der gesamten Gesellschaft entwickelt. Da auch die Viszeralmedizin im Fokus steht, sind deutliche Veränderungen im Vorgehen notwendig.

Material und Methode. Erforderliche Strukturen und Herausforderungen der Viszeralmedizin werden für notwendige Eingriffe nach Screening und bei SARS(, severe acute respiratory syndrome ")-CoV("coronavirus")2-infizierten Patienten beschrieben und die Anforderungen an die Viszeralchirurgie dargestellt. Hierfür erfolgten eine Analyse der aktuellen und themenbezogenen Literatur und die Erläuterung des Vorgehens im Krankenhaus der Grund- und Regelversorgung am Niederrhein.

Ergebnisse. Bei der Anpassung an die Krisensituation kann die Etablierung eines Systems für ein Krankenhaus in Deutschland dargestellt werden. Hierfür erfolgten unter anderem die Schaffung eines CoronaScreening-Centers am Haupteingang der Klinik und die Etablierung eines Krisenstabs für COVID-19. Spezifische interne Leitlinien wurden für das Vorgehen erstellt, erläutert, durch internationale Studien belegt und mehrfach den jeweiligen Anforderungen der Situation angepasst.

Diskussion. Die Krise ist mit deutlicher Verzögerung im Vergleich zu anderen
Ländern in Deutschland milde verlaufen. Entsprechende Vorbereitungen konnten in vielen Krankenhäusern getroffen werden, um möglichst gut vorbereitet zu sein auf schwerkranke SARS-CoV-2-infizierte Patienten auf den Intensivstationen, auf der Isolierstation, im Operationsbereich und in der Endoskopie. Der Schutz der Mitarbeiter und der Patienten ist dabei oberstes Ziel. Letztlich werden wir durch Aufarbeitung der Pandemie auch medizinische Rituale und Angewohnheiten überdenken müssen.

Schlüsselwörter

Coronavirus · Pandemie · Screening · Endoskopie · Chirurgie

\section{Challenges in visceral medicine during COVID-19. Experiences from a hospital with basic and standard care facilities}

Abstract

Background. The worldwide dissemination of the coronavirus disease 2019 (COVID-19) pandemic has become a relevant problem for the German healthcare system and the whole of society within only a few weeks. Because visceral medicine is at the focal point many adjustments in procedures are necessary. Material and methods. Necessary organizational structures and challenges in visceral medicine are described for urgent abdominal surgery after screening and for patients infected by severe acute respiratory syndrome coronavirus 2 (SARS-CoV-2). Therefore, an analysis of the current and relevant literature was performed and changes in the procedures in a hospital for basic and standard healthcare in Lower Rhine are described.

Results. This article describes the organizational structures and changes in a German hospital facing the crisis and management during the pandemic. These include establishment of a corona screening center at the hospital's main entrance and a multidisciplinary crisis management team. Specific internal guidelines were formulated for the management, confirmed by international experience and studies and regularly changed due to the requirements of the situation.
Conclusion. In comparison to other countries the crisis reached hospitals in Germany with a clear delay and a relatively mild course. In order to be prepared for severely ill patients, adequate preparations could be made to meet the challenges on intensive care units, isolation wards, operating theaters and in endoscopy. The primary goal was the safeguarding of patients and employees. In the light of the pandemic medical rituals and habits need to be reconsidered.

Keywords

Coronavirus · Pandemic · Screening .

Endoscopy · Surgery
Das im Fokus stehende SARS-CoV-2 ist, nach theoretischen Überlegungen und den Ergebnissen einer aktuellen Studie, nicht anders zu bewerten [23].

\section{Schutzausrüstung und Interventionen}

Das ärztliche Personal muss vertraut sein mit

- COVID-19 (Infektionsmodus, Symptome, Verlauf);
- Umgang mit Verdachts- und Krankheitsfällen sowie mit genesenen Patienten;

- Anwendung der persönlichen Schutzausrüstung;

- Testverfahren und deren korrekter Anwendung.

Im St. Bernhard-Hospital in KampLintfort wurde ein mehrstufiges Schulungsprogramm etabliert. Inzwischen sind die chirurgischen Kollegen trainiert worden, um die Kollegen der Medizi- nischen Kliniken beim Screening und bei der Ersttherapie von an COVID19 erkrankten Patienten (inkl. intensivmedizinische Therapie) zu entlasten. Gleichzeitig sind die internistischen und chirurgischen Mitarbeiter durch die anästhesiologischen Kollegen $\mathrm{zu}$ Fragen der Beatmung intensiv geschult worden. 


\section{Schwerpunkt}

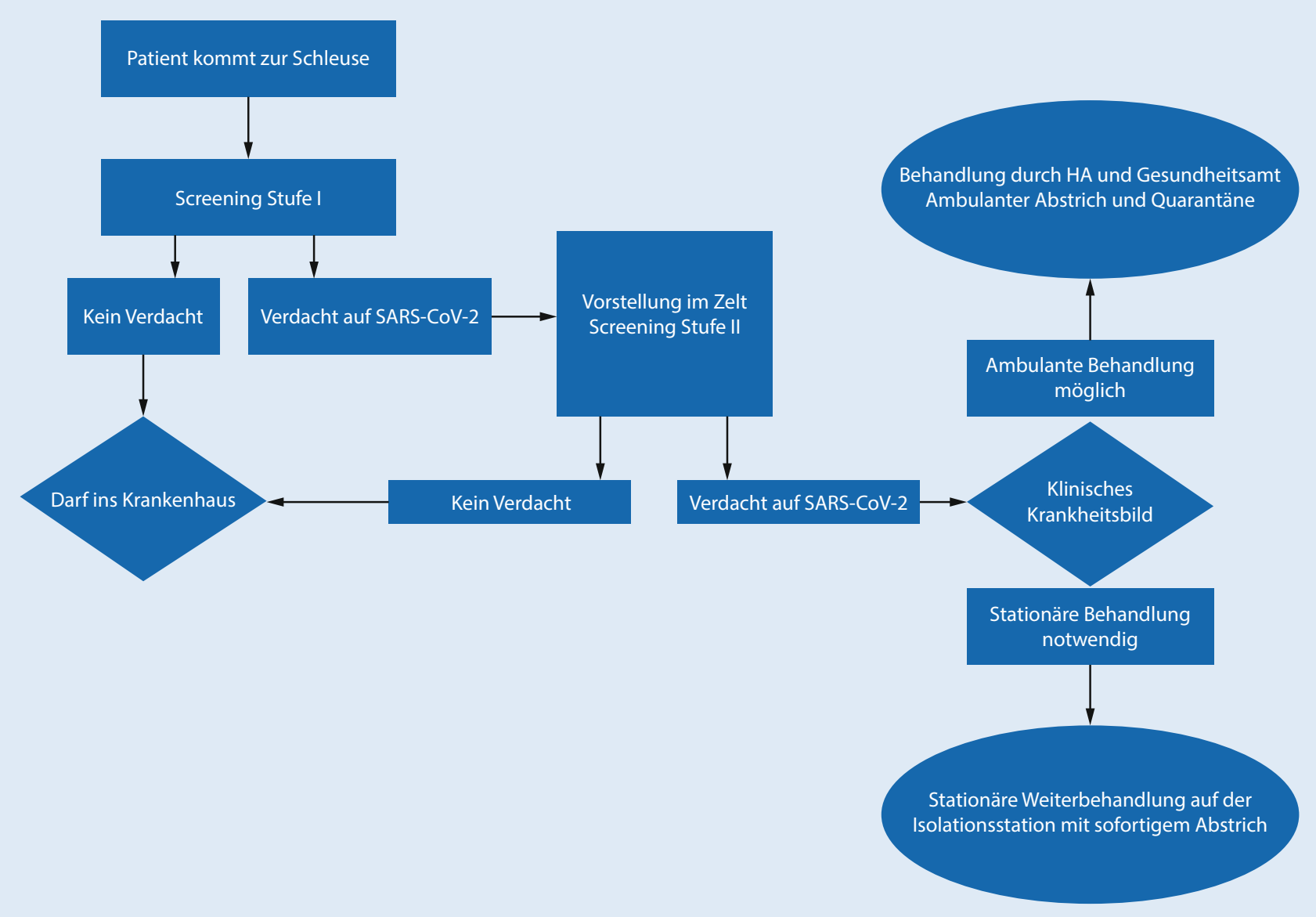

Abb. 2 A Flussschema zur Vorgehensweise im CSC (Corona-Screening-Center) Kamp-Lintfort beim Screening der Patienten und Besucher (SARS-CoV-2 "severe acute respiratory syndrome coronavirus 2", HA Hausarzt)

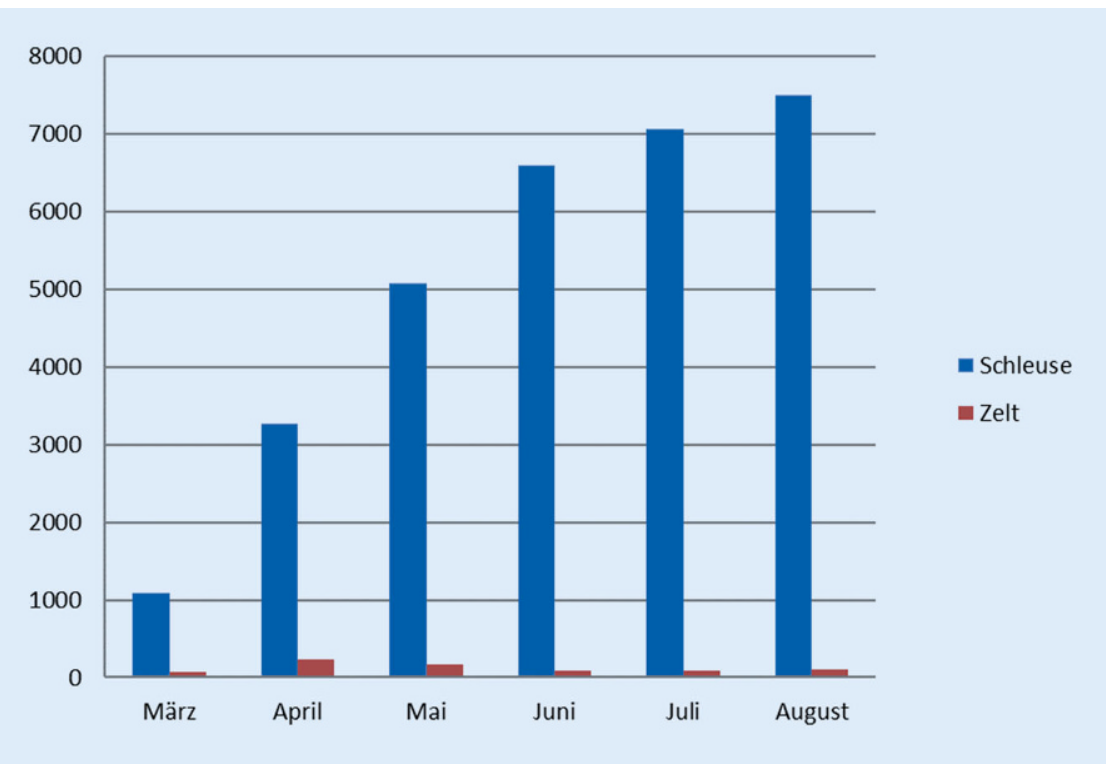

Abb. 3 A Darstellung derin derSchleuse des CSC (Corona-Screening-Center) Kamp-Lintfort gescreenten Patienten und Besucher pro Monat von März bis August 2020, zusätzlich Angabe der im Zelt bei auffälligem Befund untersuchten Patienten

\section{Besonderheiten für die Viszeralmedizin}

Ziel der Arbeit ist es, die Durchführbarkeit aller medizinisch notwendigen Maßnahmen darzustellen, aber besondere Schutzmaßnahmen und Handlungsstrategien für die Viszeralmedizin sicherzustellen. Diese müssen dazu beitragen, zum einen die Pandemie zu begrenzen und zum anderen die Mitarbeiter, mögliche Besucher und die Gesamtheit der Patienten zu schützen. Besonderheiten für die Behandlung im Umfeld der interventionellen und operativen Therapie werden im Folgenden erörtert.

Die Mitarbeiter des Gesundheitssystems, besonders die Teams der Viszeralmedizin, sind zum einen selbst gesundheitlichen Risiken und zum anderen Herausforderungen in der Behandlung COVID-19-positiver Patienten ausgesetzt. Aus dem Umgang mit Patienten, 


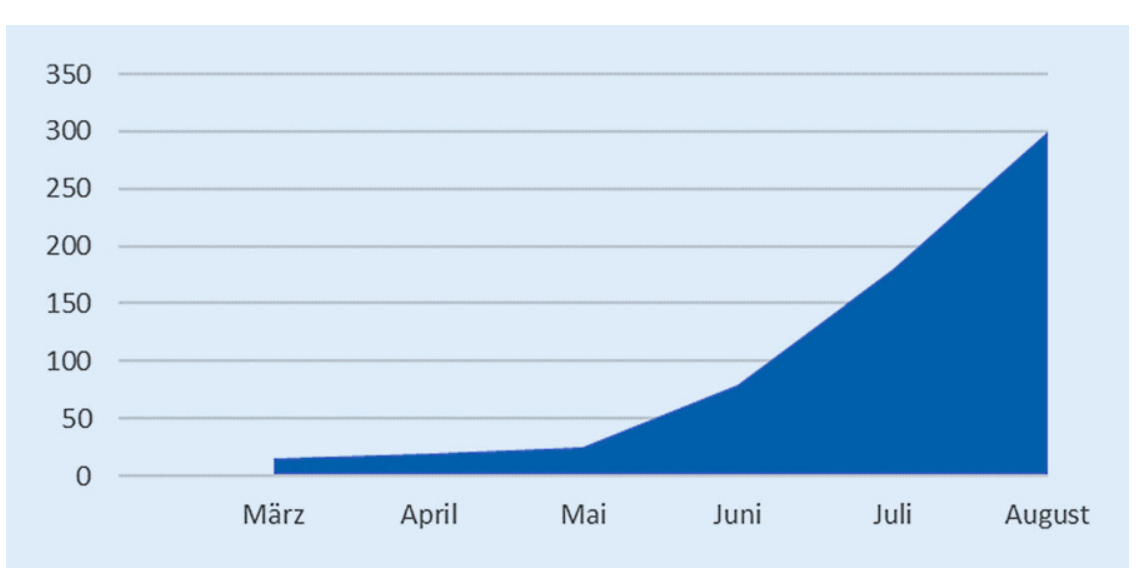

Abb. 4 \ Darstellung der Entwicklung der Besucherzahl pro Monat seit Einführung des CSC (CoronaScreening-Center) Kamp-Lintfort seit Mitte März bis Ende August unter Lockerung der bestehenden Besucherbeschränkungen

die einen bloßen Verdachtsfall darstellen oder einer sog. Hochrisikogruppe entstammen, ergibt sich eine weitere Problematik.

\section{》) Viszeralmedizinische Notfälle durch das Virus sind bisher als seltene Komplikation anzusehen}

Viszeralmedizinische Notfälle durch das Virus sind bisher als seltene Komplikation anzusehen [13]. In der Vergangenheit sind Viruserkrankungen durch viszeralmedizinische Komplikationen aber bereits problematisch geworden (etwa Kolonischämien, toxische Enteritiden durch H1N1, SARS-CoV und MERS(„Middle East respiratory syndrome")-(CoV), sodass bei Notfallpatienten auch an diese Pathogenese gedacht werden muss [17, 18].

In den letzten Monaten haben andere Gesundheitssysteme etwa in China, in den USA, in Italien und in Spanien bei extrem hohem (und unerwartetem) Patientenaufkommen Krisen erlebt mit teilweise sehr hohen Mortalitätsraten bis hin zur Notwendigkeit von ethisch fraglichen Triagierungen [23].

Kollegen aus Norditalien berichten über kriegsähnliche Zustände mit sehr hohen Mortalitäten (Durchschnitt in Italien: $\leq 10 \%$ ), andere wiederum über eine große medizinische Erfolgsrate, z. B. in Singapur [16, 20].

Einige der Erfahrungen der internationalen Kollegen können nach Deutsch- land übertragen werden. Unter den in Deutschland gültigen politischen Vorgaben sind die elektive Endoskopie und das Operationsprogramm über viele Wochen massiv reduziert worden. Nur Notfälle und besonders dringliche Patienten sollten behandelt werden. Alle Entwicklungen und auch politische Vorgaben verlaufen dynamisch, teilweise nicht gut vorhersehbar, sodass das Vorgehen tagesaktuell reevaluiert werden muss.

Betrachtet man das Krankenhaus als Wirtschaftsunternehmen, entsteht möglicherweise ein finanzieller Engpass durch freie vorgehaltene Betten, die für keine andere medizinische Leistung zur Verfügung stehen. Ein enger Austausch sowohl der ärztlichen Kollegen untereinander als auch mit den übergeordneten Behörden und der Krankenhausleitung ist deshalb erforderlich. Im Prinzip haben daher alle Krankenhäuser ein Krisenteam zur raschen Anpassung wechselnder Gegebenheiten gegründet. Die unserem Krankenhaus zur Verfügung gestellte Ausfallentschädigung in Höhe von $560 €$ je nicht belegtes Krankenhausbett konnte kurzfristig Liquidationsprobleme verhindern, langfristige Folgen sind jedoch noch nicht absehbar.

\section{Behandlungsplanung und Indikationsstellung}

Muss ein Eingriff nach Indikationsstellung durch einen Facharzt durchgeführt werden, müssen besondere Aspekte sowohl zum Schutze der Patienten als auch zur Minimierung von Risikofaktoren für das behandelnde Team beachtet werden [2].

Elektive Operationen, endoskopische Eingriffe und auch elektive ambulante Behandlungen wurden ab Mitte März 2020 bis Ende April verschoben. Notfalleingriffe und dringliche Operationen wurden aber weiter geplant und durchgeführt. Erläuterungen hierzu gab etwa eine Publikation im Deutschen Ärzteblatt [9]. In unserer Beobachtung sind Patienten auch im Herbst 2020 noch sehr zurückhaltend und versuchen, Krankenhausaufenthalte zu vermeiden.

Neben den vitalen Notfällen in der Gastroenterologie und in der Chirurgie sind aus medizinischer Sicht und aufgrund ethischer Betrachtungen onkologische Behandlungen, aber auch andere Therapien wie Transplantationen und endokrine Eingriffe als dringlich zu werten, und diese erfolgten auch während des sog. Lockdowns. Gleiches gilt für therapeutische Endoskopien etwa bei Blutungen, dringliche endoskopisch retrograde Cholangiopankreatikographien (ERCP) oder anderweitige Interventionen, die zur Behandlung akuter Symptome indiziert sind.

\section{Radiologische Bildgebung im Rahmen der Behandlungs- planung}

Die Notwendigkeit adäquater Bildgebung zur Indikationsstellung und Therapieplanung bleibt auch unter Pandemiebedingungen bestehen. Dies bedeutet, dass die Indikationen zu radiologischen Untersuchungen im Kontext begrenzter Ressourcen (Personal, persönliche Schutzausrüstung) und der verfügbaren Großgeräte gestellt werden. Dennoch muss die notwendige bildgebende $\mathrm{Di}$ agnostik zeitnah und in leitlinienkonformer Qualität erfolgen (• Abb.5). Das kann im Einzelfall den Umstieg auf alleinige Ultraschalldiagnostik, aber auch den Verzicht auf (weniger aussagefähige) konventionelle Diagnostik und direkte $\mathrm{CT}$ (Computertomographie)Bildgebung bedeuten. Aufgrund der häufig fehlenden intensivmedizinischen Ausrüstung muss die Verwendung der Magnetresonanztomographie (MRT) auf 


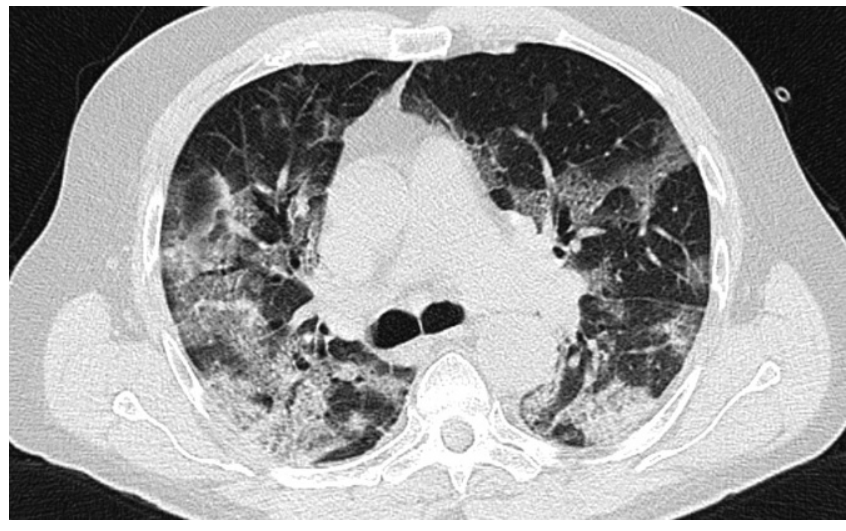

Abb. $5<\ln \operatorname{der} C T$ (Computertomographie) des Thorax zeigen sich typische Befunde für eine SARS (, severe acute respiratory syndrome $\left.{ }^{\prime \prime}\right)-\operatorname{CoV}($, coronavirus")-2-Infektion. Die sichtbaren Milchglasinfiltrate weisen auf Virusbefall und eine hierdurch bedingte Pneumonie hin

klinisch stabile Patienten reduziert werden. Die empfohlenen Leitlinien („best practice") für die CT-Bildgebung erfordern eine angepasste Personalplanung auch in der Radiologie [12, 19, 21].

\section{》) Die Notwendigkeit adäquater Bildgebung bleibt auch unter Pandemiebedingungen bestehen}

Das reduzierte viszeralmedizinische Programm soll dazu führen, dass sich das Virus unter den im Krankenhaus befindlichen Personen (Patienten, wenige Besucher und sämtliche Berufsgruppen) nicht verbreitet. Dadurch kommt es zu einer Einsparung von limitierten Ressourcen wie persönlicher Schutzausrüstung, Krankenhausbetten und insbesondere intensivmedizinischen Kapazitäten. Sowohl die medizinischen als auch die ökonomischen Folgen sind für das gesamte System langfristig nicht abzusehen.

\section{Empfehlungen für Interven- tionen und perioperatives Vorgehen}

In Krankenhäusern, die dringliche Interventionen für mit SARS-CoV-2 infizierte Patienten anbieten, sollte ein speziell vorbereiteter Behandlungsraum vorgehalten werden. Dieser muss dazu geeignet sein, eine Ausbreitung von SARS-CoV-2 auf die übrigen Bereiche zu unterbinden. Alle beteiligten Personen müssen umfassend in allen Aspekten der SARS-CoV-2-Pandemie geschult sein und mit Schutzklei- dung arbeiten. Die internationalen Erfahrungen und Empfehlungen sind dabei für die Umsetzung sehr hilfreich [2, $3,5,6,8,15,16,23]$.

Folgende Schlüsselelemente sind besonders wichtig:

1. Identifikation und Definition spezifischer Behandlungsräume für alle COVID-19-Patienten, unabhängig von der Fachrichtung. Dieser Raum sollte außerhalb der hochfrequentierten notfallmäßigen Operationsbereiche (z.B. Trauma- oder Tumorzentrum) lokalisiert sein.

2. Analog zu dem hygienekonformen Vorgehen bei multiresistenten Keimen muss dieser Bereich von nicht erforderlichen Materialien leergeräumt sein.

3. Der Patient sollte in einer abgetrennten isolierten Narkosevorbereitung in einem mit Unterdruck versehenen Raum eingeleitet werden und über eine Schleuse bereits intubiert und beatmet transportiert werden. Steht eine solche gesondert klimatisierte Einheit nicht zur Verfügung, ist der Patient im klimatisierten Behandlungsraum/Operationssaal unter Verwendung adäquater Schutzausrüstung mit FFP3(,filtering face piece 3“)-Maske im Sinne einer RSI („rapid sequence induction") durch ein 2-Personen-Team unter Verwendung eines Videolaryngoskops einzuleiten. Das weitere Team sollte nebst „Springer" der Anästhesie vor dem Operationssaal verbleiben und erst 15 min nach Intubationsvorgang unter Verwendung der persönlichen Schutzausrüstung den Raum betreten, wobei der „Springer“ des
Anästhesiefachpersonals weiter zur Verfügung steht.

4. Der direkte Bereich des Operationssaals und des Endoskopieraums muss nach außen mit auffälligen Markern gekennzeichnet sein, um zu gewährleisten, dass nur das notwendige Personal den Bereich betritt. Poster zum An- und Ausziehen der geeigneten Schutzkleidung sollen gut sichtbar für das Personal in den Waschräumen und im Schleusenraum vorhanden sein.

5. Persönliche Sachen (wie Handys und Stifte) dürfen nicht mit in den Raum gebracht werden.

6. Alle Einmalartikel müssen am Ende der Operation verworfen werden.

7. Ein speziell für diesen Behandlungsraum/Operationssaal abgestellter und aufgeklärter „Springer“ muss alle erforderlichen Materialien bringen.

8. Wenn möglich, soll der Patient noch im Behandlungsraum/Operationssaal erwachen und extubiert werden, sodass er zur anschließenden postoperativen Betreuung auf die dafür vorgesehene Isolierungsstation verbracht werden kann. Der Transportweg des Patienten zum und aus dem Operationstrakt muss dabei klar definiert und möglichst kurz sein.

9. Jede Klinik sollte einen COVID-19beauftragten Arzt definieren, der an das Kriseninterventionsteam angeschlossen ist und über die von extern (z. B. durch das RKI) an das Krankenhaus herangetragenen Informationen verfügt. In der abgebildete- Klinik ist der beauftragte Kollege gleichzeitig der koordinierende Arzt des COVIDScreening Centers (CSC), welches als Provisorium vor dem Krankenhaus aufgebaut wurde (• Abb. 1).

\section{Fazit für die Praxis}

- Bereits die letzten Monate haben gezeigt, dass das deutsche Gesundheitssystem sich trotz Widerständen rasch auf eine Pandemie und drastisch geänderte politische Vorgaben einstellen kann.

- Eine klare Strategie muss sowohl unser eigenes Personal schützen als auch die Ausbreitung von COVID-19 
(",coronavirus disease 2019“) innerhalb des Krankenhauses verhindern. - Darüber hinaus fordert die Situation nicht nur viele Epidemiologen, Virologen und weitere Wissenschaftler und Ärzte heraus, sondern zwingt uns zum Überdenken medizinischer Rituale und Angewohnheiten.

- Die wirtschaftlichen Auswirkungen auf unser Gesundheitssystem sind noch unklar und auch von der Dauer und dem weiteren Verlauf der Pandemie abhängig.

\section{Korrespondenzadresse}

\section{Dr. T. Heuer}

Medizinische Klinik I, St. Bernhard-Hospital

Bürgermeister-Schmelzing-Str. 90,

47475 Kamp-Lintfort, Deutschland theodor.heuer@st-bernhard-hospital.de

\section{Einhaltung ethischer Richtlinien}

Interessenkonflikt. M. Banysch, T. Heuer, G. Frings, H. Kühl, S. Bochlogyros und G. M. Kaiser geben an, dass kein Interessenkonflikt besteht.

Für diesen Beitrag wurden von den Autoren keine Studien an Menschen oder Tieren durchgeführt. Für die aufgeführten Studien gelten die jeweils dort angegebenen ethischen Richtlinien.

\section{Literatur}

1. Alp E, Bijl D, Bleichrodt RP, Hansson B, Voss A (2006) Surgical smoke and infection control. J Hosp Infect 62:1-5

2. Brindle M, Gawande A (2020) Managing COVID-19 in surgical systems. Ann Surg 272:e1-e2

3. Chen X, Liu Y, Gong Y (2020) Perioperative management of patients infected with the novel Coronavirus. Anesthesiology 132:1307-1316

4. Fiedler M, Kaiser GM, Paul A (2010) Severe H1N1 infection in a pediatric transplant recipient treated with intravenous zanamivir: efficiency and complications. Transplantation 90:223-224

5. Greenland JR, Michelow MD, Wang L, London MJ (2020) COVID-19 Infection Implications for Perioperative and Critical Care. Physicians Anesthesiol 132:1346-1361

6. Grundmann $\mathrm{H}$, Donker $\mathrm{T}$, Hengel $\mathrm{H}$, Bürkle $\mathrm{H}$, Hammer T, Wenz F, Kern WV (2020) Universelles Aufnahmescreening - Eintragungsrisiko beurteilen. Dtsch Arztebl 117:B1378

7. KwakHD, Kim SH, Seo YS, Song KJ (2016) Detecting Hepatitis B Virus in Surgical Smoke emitted during Laparoscopic Surgery. Occup Environ Med 73:857-863

8. Lancaster EM, Sosa JA, Sammann A (2020) Rapid Response of an Academic Surgical Department to the COVID-19 Pandemic: Implications for Patients, Surgeons, and the Community. J Am Coll Surg 230:1064-1073
9. Lenzen-Schulte M (2020) COVID-19: Chirurgie in Zeiten der Pandemie. Dtsch Arztebl 117:A-940 / B-793

10. Li Cl, Pai JY, Chen CH (2020) Characterisation of smoke generated during the use of surgical knife in laparotomy surgeries. J Air Waste Manag Assoc 70:324-332

11. Lynch JB, Davitkov P, Anderson DJ (2020) Infectious diseases Society of America guidelines on infection prevention for health care personnel caring for patient with suspected or known COVID-19. Clin Infect Dis. https://doi.org/10.1093/cid/ciaa1063

12. Nakajima $K$, Kato $H$, Yamashiro T, Izumi $T$, Takeuchi I, Nakajima H, Utsunomiya D (2020) COVID-19 pneumonia: infection control protocol inside computed tomography suites. Jpn J Radio 38:391-393

13. Norsa $L$, Valle C, Morotti D, Bonaffini PA, Indriolo $A$, Sonzogni A (2020) Intestinal ischemia in the COVID-19 era. Dig Liver Dis 52(10):1090-1091. https://doi.org/10.1016/j.did.2020.05.030

14. Robert-Koch-Institut Robert-Koch-Institut. https://www.rki.de/DE/Content/InfAZ/N/ Neuartiges_Coronavirus/Fallzahlen.html. Zugegriffen:3. Sept. 2020

15. Thomas-Rüddel $D$, Winning J, Dickmann $P$ (2020) Coronavirus disease 2019 (COVID-19): Update für Anästhesisten und Intensivmediziner. Anaesthesist 69:225-235

16. Li LK, Ang LS, Foong TW (2020) What we do when a COVID-19 patient needs an operation: operating room preparation and guidance. Can J Anesth 67:756-758

17. Vernadakis S, Adamzik M, Heuer M (2010) Hemikolektomie bei Kolonischämie. Chirurg 81:841-845

18. Vernadakis S, Heuer M, Brackmann C, Paul A, Kaiser GM (2012) Intestinal Ischemia Secondary to H1N1 Influenza. Am Surg 78:144-146

19. Wan YL, Schoepf UJ, Wu CC, Giovagnoli DP, Wu MT, Hsu HH, Chang YC, Yang CT, Cherng WJ (2020) Preparedness and best practice in radiology department for COVID-19 and other future pandemics of severe acute respiratory infection. Radiology 296:E106-E112

20. World Health Organisation https://www.who.int/ emergencies/diseases/novel-coronavirus-2019/ situation-reports. Zugegriffen: 3. Sept. 2020

21. Zanardo M, Martini C, Monti CB, Cattaneo F, Ciaralli C, Cornacchione P, Durante S (2020) Management of patients with suspected or confirmed COVID19 , in the radiology department. Radiography 23(3):264-268. https://doi.org/10.1016/j.radi. 2020.04.010

22. Zhao $Y$, Xiang C, Wang S, Peng C, Zou Q, $\mathrm{Hu} J$ (2020) Radiology department strategies to protect radiologic technologists against COVID19: Experience from Wuhan. Eur JRadiol 127:108

23. Zheng MH, Boni L, Fingerhut A (2020) Minimally invasive surgery and the novel coronavirus outbreak: lessons learned in China and Italy. Ann Surg 272:e5-e6
Indikationsqualität in der Viszeralchirurgie

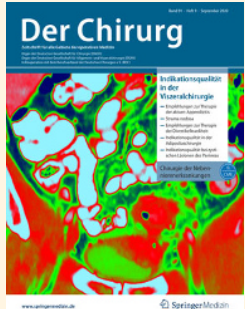

Die richtige und angemessene Indikationsstellung zu einem operativen Eingriff bestimmt ganz wesentlich dessen chirurgische Qualität. Im Leitthema in Der Chirurg 09/2020 werden deshalb häufige chirurgische Eingriffe in den Mittelpunkt gerückt, deren Durchführungsfrequenz sich immer wieder einer kritischen Diskussion ausgesetzt sieht. Chirurgische Indikationen müssen sich an evidenzbasierten Grundlagen und Standards orientieren, zu denen klinische Studienergebnisse und Leitlinien zählen. Darüber hinaus spielt aber auch die individuelle ärztliche Erfahrung eine wesentliche Rolle zur Entscheidungsfindung.

Wir bieten Ihnen mit diesem Heft eine hilfreiche aktuelle Übersicht für die tägliche Indikationsstellung bei wichtigen viszeralmedizinischen Krankheitsbildern.

- Empfehlungen zur Therapie der akuten Appendizitis

- Struma nodosa

- Empfehlungen zur Therapie der Divertikelkrankheit

- Indikationsqualität in der Adipositaschirurgie

- Indikationsqualität bei zystischen Läsionen des Pankreas

\section{Suchen Sie noch mehr zum Thema?} Mit e.Med - den maßgeschneiderten Fortbildungsabos von Springer Medizin - haben Sie Zugriff auf alle Inhalte von SpringerMedizin.de. Sie können schnell und komfortabel in den für Sie relevanten Zeitschriften recherchieren und auf alle Inhalte im Volltext zugreifen.

Weitere Infos zu e.Med finden Sie auf springermedizin.de unter „Abos" 